\title{
CONVENTIONS and ABBREVIATIONS USED
}

$A T L=$ The Athenian Tribute Lists. B. D. Meritt, H. T. Wade-Gery, and M. F.

McGregor. Volume 3. Princeton. I950.

$A R V=$ Attic Red-Figure Vase Painters. J. D. Beazley. Second edition. Oxford. 1963. Bekker, Anec. Graec. = Anecdota Graeca 3 vols. I. Bekker. Berlin. I8I4-I84I.

$D F A=$ The Dramatic Festivals of Athens. A Pickard-Cambridge. Second edition. I968. Revised with supplement by J. Gould and D. M. Lewis. Oxford. 1988.

D-K = Die Fragmente der Versokratiker. H. Diels and W. Kranz (eds.). Sixth edition. Zurich. 1952.

Et. Magn. = Etymologicum Magnum. F. Sylburg. Leipzig. I8I6.

FGrHist $=$ Fragmente Griechischer Historiker. F. Jacoby. Berlin and Leiden. 1923-1958.

$I G=$ Inscriptiones Graecae. Berlin. I873-.

$\mathrm{K}-\mathrm{A}=$ Poetae Comici Graeci. R. Kassel and C. Austin (eds.). Berlin. 1983-.

$M L=A$ Selection of Greek Historical Inscriptions. R. Meiggs and D. Lewis. Revised edition. Oxford. I969.

$M N C=$ Monuments Illustrating New Comedy. T. B. L. Webster. Volumes I-2. Third edition, revised by J. R. Green and A. Seeberg. (BICS Supplement 50). London. 1995 .

MTS $=$ Monuments Illustrating Tragedy and Satyr Play. T. B. L. Webster. Second edition. London. 1968.

Osborne, Naturalization $=$ Naturalization in Athens. M. J. Osborne. 4 volumes. Brussels. I98I-I983.

P.A. = Prosopographia Attica .2 volumes. J. Kirchner. Berlin. I90I-I903.

PHibeh = The Hibeh Papyri II. E. G. Turner (ed.). London. I955.

$P M G=$ Poetae Melici Graeci. D. L. Page (ed.). Oxford. 1962.

POxy = The Oxyrhynchus Papyri. B. P. Grenfell et al. (eds.). London. I898-. SEG $=$ Supplementum Epigraphicum Graecum. Leiden. I923-. 
$\operatorname{Tr} G F=$ Tragicorum Graecorum Fragmenta. R. Kannicht, S. Radt, B. Snell (eds.).

Göttingen. 197I-2004.

\section{IOURNAL ABBREVIATIONS}

\begin{tabular}{|c|c|}
\hline$A H B$ & Ancient History Bulletin \\
\hline$A I O N$ & Annali dell'Istituto Universitario Orientali di Napoli \\
\hline AJA & American Journal of Archaeology \\
\hline AJAH & American Journal of Ancient History \\
\hline$A J P$ & American Journal of Philology \\
\hline$A M$ & $\begin{array}{l}\text { Mitteilungen des Deutschen Archäologischen Instituts, Athenische } \\
\text { Abteilung }\end{array}$ \\
\hline AncSoc & Ancient Society \\
\hline ArchDelt & Archaiologikon Deltion \\
\hline$A S N P$ & Annali della Scuola Normale Superiore di Pisa \\
\hline$A W$ & Ancient World \\
\hline$B I C S$ & Bulletin of the Institute of Classical Studies of the University of London \\
\hline$B S A$ & Annual of the British School at Athens \\
\hline ClAnt & Classical Antiquity \\
\hline$C J$ & Classical Journal \\
\hline$C \mho M$ & Classica et Mediaevalia \\
\hline$C P$ & Classical Philology \\
\hline$C Q$ & Classical Quarterly \\
\hline$C R$ & Classical Review \\
\hline$G \dot{U} R$ & Greece and Rome \\
\hline GRBS & Greek, Roman, and Byzantine Studies \\
\hline$H S C P$ & Harvard Studies in Classical Philology \\
\hline ICS & Illinois Classical Studies \\
\hline$J D A I$ & Jahrbuch des Deutschen Archäologischen Instituts \\
\hline JHS & Journal of Hellenic Studies \\
\hline PCPS & Proceedings of the Cambridge Philological Society \\
\hline QUCC & Quaderni urbinati di cultura classica \\
\hline$R E$ & Real-Encyclopädie der classischen Alterthumswissenschaft \\
\hline$R E G$ & Revue des études grecques \\
\hline$S M S R$ & Studi e materiali di storia delle religioni \\
\hline TAPA & Transactions of the American Philological Association \\
\hline$T R I$ & Theatre Research International \\
\hline WJA & Würzburger Jahrbücher für die Altertumswissenschaft \\
\hline WS & Wiener Studien \\
\hline$Z P E$ & Zeitschrift für Papyrologie und Epigraphik \\
\hline
\end{tabular}


Authors in square brackets indicate that the authorship is uncertain for a work that has been traditionally ascribed to the author in question.

References to the scholia are indicated by $\Sigma$.

I have not been consistent with the transliteration of Greek names and terms, but I have tried to maintain a certain logic in my usage. Those names and terms with entries in the Oxford Classical Dictionary appear as they do in that reference work. Readers will thus find the well-known "Sophocles" and the relatively unknown "Nikostratos."

Translations are my own unless noted otherwise.

ABBREVIATIONS USED FOR ANCIENT AUTHORS

$\begin{array}{llll}\text { Ael. } & \text { Aelian } & \text { Is. } & \text { Isaeus } \\ \text { Aesch. } & \text { Aeschylus } & \text { Isoc. } & \text { Isocrates } \\ \text { Aeschin. } & \text { Aeschines } & \text { Lib. } & \text { Libanius } \\ \text { AG } & \text { Aulus Gellius } & \text { Luc. } & \text { Lucian } \\ \text { Andoc. } & \text { Andocides } & \text { Lyc. } & \text { Lycurgus } \\ \text { Ar. } & \text { Aristophanes } & \text { Lys. } & \text { Lysias } \\ \text { Arist. } & \text { Aristotle } & \text { Men. } & \text { Menander } \\ \text { Ath. } & \text { Athenaeus } & \text { Paus. } & \text { Pausanias } \\ \text { Dem. } & \text { Demosthenes } & \text { Philoch. } & \text { Philochorus } \\ \text { Din. } & \text { Dinarchus } & \text { Pl. } & \text { Plato } \\ \text { Diod. Sic. } & \text { Diodorus Siculus } & \text { Plaut. } & \text { Plautus } \\ \text { Diog. Laert. } & \text { Diogenes Laertius } & \text { Plut. } & \text { Plutarch } \\ \text { Eup. } & \text { Eupolis } & \text { Soph. } & \text { Sophocles } \\ \text { Eur. } & \text { Euripides } & \text { Theocr. } & \text { Theocritus } \\ \text { Harp. } & \text { Harpocration } & \text { Theophr. } & \text { Theophrastus } \\ \text { Hdt. } & \text { Herodotus } & \text { Theopomp. } & \text { Theopompus } \\ \text { Hesych. } & \text { Hesychius } & \text { Thuc. } & \text { Thucydides } \\ \text { Hyp. } & \text { Hyperides } & \text { Xen. } & \text { Xenophon }\end{array}$


THIS PAGE INTENTIONALLY LEFT BLANK 